\title{
JOINT EUROPA MISSION (JEM) \\ A MULTISCALE, MULTI-PLATFORM MISSION TO CHARACTERIZE EUROPA'S HABITABILITY AND SEARCH FOR EXTANT LIFE
}

\author{
A White Paper prepared for the \\ NAS 2023-2032 Decadal Survey for Planetary Science and Astrobiology \\ August 15th, 2020
}

Michel Blanc(1), Olga Prieto-Ballesteros(2), Nicolas André(1), Javier Gomez-Elvira(2), Geraint Jones(3), Veerle Sterken(4,5), William Desprats(1,6), Leonid I. Gurvits(7,16,38), Krishan Khurana(8), Georges Balmino(39), Aljona Blöcker(9), Renaud Broquet(10), Emma Bunce(11), Cyril Cavel(10), Gaël Choblet(12), Geoffrey Colins(13), Marcello Coradini(14), John Cooper(15), Dominic Dirkx(16), Dominique Fontaine(40), Philippe Garnier(1), David Gaudin(6), Paul

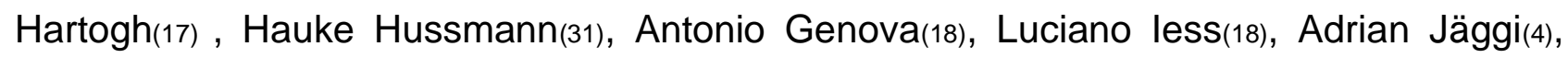
Sascha Kempf(19), Norbert Krupp(17), Luisa Lara(20), Jérémie Lasue(1), Valéry Lainey(21), François Leblanc(22), Jean-Pierre Lebreton(23), Andrea Longobardo(24), Ralph Lorenz(25), Philippe Martins(26), Zita Martins(27), Jean-Charles Marty(41), Adam Masters(28), David Mimoun(6), Ernesto Palumba(33), Victor Parro(2), Pascal Regnier(10), Joachim Saur(29), Adriaan Schutte(30), Edward C. Sittler(15), Tilman Spohn(31), Ralf Srama(42), Katrin Stephan(31), Károly Szegő(32), Federico Tosi(33), Steve Vance(34), Roland Wagner(31), Tim Van Hoolst(35), Martin Volwerk(36), Jan-Erik Wahlund(41), Frances Westall(37), Peter Wurz(4)

Affiliations: (1)IRAP, France ; (2)INTA-CAB, Spain; (3)MSSL/UCL, UK ; (4)University of Bern, Switzerland; (5)ETH Zürich, Switzerland; (6)ISAE, France; (7)Joint Institute for VLBI ERIC, Dwingeloo, The Netherlands; (8)UCLA, USA ; (9) Royal Institute of Technology KTH, Sweden, (10)Airbus D\&S, France, (11)Univ. Leicester, UK; (12)LPG, Univ. Nantes, France; (13)Wheaton College, USA; (14)CSEO, USA; (15)GSFC, USA; (16)TU Delft, The Netherlands; (17)MPS, Germany; (18)Univ. Roma La Sapienza, Italy; (19)LASP, Univ. Colorado, USA; (20)IAA, Spain; (21)IMCCE, France; (22)LATMOS, France; (23)LPC2E, France; (24)IAPS, Italy; (25)APL/JHU, USA ; (26)Télécom ParisTech, France ; (27) CQE, IST, Portugal; (28)Imperial College, UK; CBM, France; (29)University of Cologne, Germany; (30)SKA Organisation, UK; (31)DLR, Germany; (32)WIGNER Institute, Hungary; (33)IAPS, Italy; (34)Jet Propulsion Laboratory, USA; (35)ROB, Belgium; (36)IWF, Austria; (37)CBM-CNRS, France; (38)CSIRO Astronomy and Space Science, Marsfield, NSW, Australia; (39)GET, Toulouse, France; (40)LPP, Palaiseau, France; (41)CNES-GRGS, Toulouse, France; (41)IRF, Uppsala, Sweden; (42)University of Stuttgart, Germany; 


\section{Executive Summary}

In this White Paper we propose that NASA works with ESA and other potentially interested international partners to design and fly jointly an ambitious and exciting planetary mission to characterize Europa's habitability and search for bio-signatures in the environment of Europa (surface, subsurface and exosphere). By choosing the Jupiter system as our destination, we can build on the advanced understanding of this system which the missions preceding JEM will provide: improved understanding of its origin and formation (Juno), of its evolutionary mechanisms (JUICE) and a preliminary comparative understanding of the habitability of its different moon by ESA's JUICE and NASA's Europa Clipper. We propose the following overarching goals for the JEM mission:

Understand Europa as a complex system responding to Jupiter system forcing, characterize the habitability of its potential biosphere, and search for life in its surface, sub-surface and exosphere.

We describe the observation strategy we propose to address them, which combines three scientific measurement sequences: 1- measurements on a high-latitude, low-latitude Europan orbit providing a continuous and global mapping of planetary fields (magnetic and gravity) and of the neutral and charged environment during a period of three months; 2- in-situ measurements to be performed at the surface, using a soft lander operating during 35 days, focusing on the search for bio-signatures at the surface and sub-surface using advanced analytical techniques in the solid and liquid phases, and the operation of a surface geophysical station whose measurements will complement those of the orbiter; 3- measurements of the chemical composition of the very low exosphere, to be performed near the end of the mission during the final descent phase.

These observation sequences will be performed by two science platforms: a soft lander to perform all scientific measurements at the surface and sub-surface and an orbiter to perform the orbital and descent sequences. In this concept, the orbiter will carry the lander stack from the Earth to a Europan orbit on which it will release it for its descent, before providing the data relay during the 35 days of lander operations. Using its instrument platform, the carrier will perform science operations during the relay phase, before moving to its final Europan science orbit for three months.

For the orbiter scientific platform, our proposed payload suite includes seven well-proven instruments to characterize the planetary fields and the plasma, neutrals and dust environment, fitting within our projected allocated mass.

Our lander science platform is composed of a geophysical station and of two complementary astrobiology facilities dedicated to bio-signature characterization experiments operating respectively in the solid and in the liquid phases. The design and development of the liquid phase laboratory, called AWL for "Astrobiology Wet Laboratory", could be a specific European contribution to the surface science platform. The two astrobiology facilities will be fed by a common articulating arm operating at the platform level that will collect the samples at the surface or sub-surface and will deliver them to the analytical facilities.

To fly JEM while making this mission an appealing and affordable joint exploration venture for both ESA and NASA, we propose an innovative distribution of roles; ESA would design and provide the carrier-orbiter-relay platform while NASA would provide an SLS launcher and the lander stack. We showed that this ESA contribution is technically possible, most likely as an M- or L-class mission, taking advantage of a double heritage of European developments: the JUICE spacecraft for the JEM orbiter avionics, and an adaptation of the ORION ESM bus to the specific needs of JEM for its structure. Following this approach, JEM will be a major exciting joint venture to the outer solar system of ESA and NASA, working together to share one of the most exciting scientific endeavors of the XXIst century: to search for life beyond our own planet. A more comprehensive description of the JEM mission concept, science objectives, scientific payload and mission scenario is given in [1], [2]. 


\section{Introduction.}

This White Paper echoes a proposition submitted a year ago to ESA's call for ideas for the "Voyage 2050" science planning exercise [1]. As the JEM mission concept is based on a joint endeavor between NASA and ESA, the JEM team wishes to submit it with the same content to the NAS Decadal Survey to feed discussions of the next cycle of NASA mission planning. Actual implementation of this concept relies entirely on its adoption by both ESA and NASA, following recommendations by their relevant advisory bodies. A detailed description of JEM can be found in [2].

\section{JEM science objectives.}

The JEM science plan and overarching goals will take maximum advantage of the achievements of previous missions to the Jupiter System (Juno, JUICE, Europa Clipper) to optimize its complementarity with them while focusing on the search for life at Europa. JEM will fly for the first time a Europa orbiter and a lander to Europa's surface to perform unique astrobiology and geophysics/geochemistry science and address Its Overarching Goal:

"Understand Europa as a complex system responding to Jupiter system forcing, characterize the habitability of its potential biosphere, and search for life in the surface, sub-surface and exosphere."

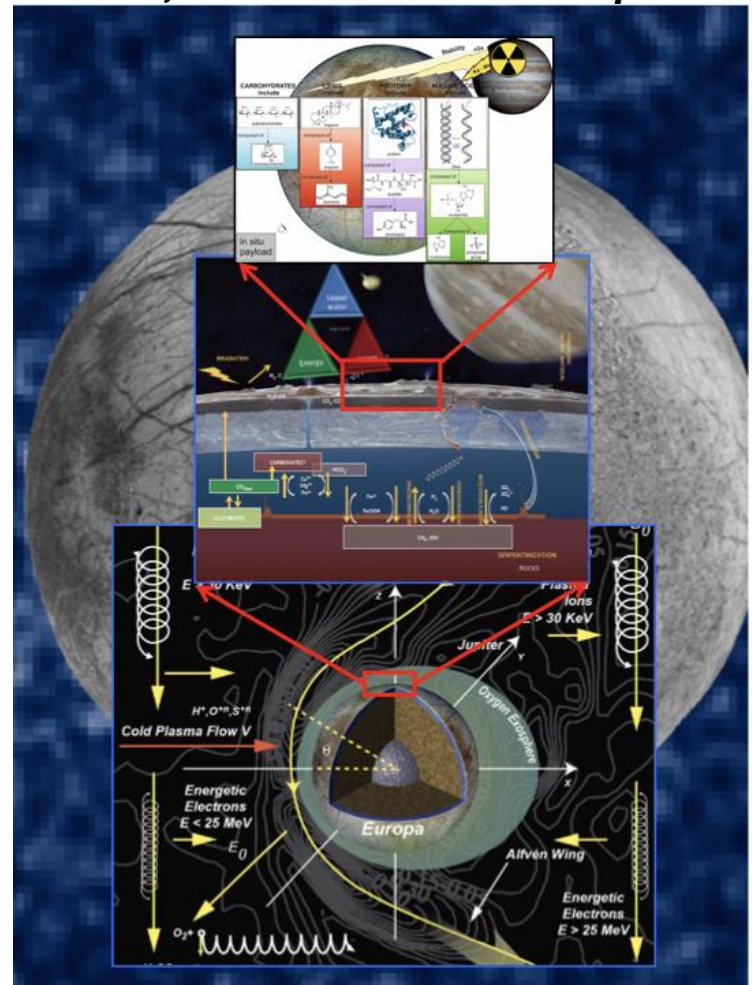

Figure 1: This logical chart of our Science Plan shows the three successive scales investigated by JEM, from bottom upwards: (1) the global Europa, a complex system responding to the two main types of Jovian forcing, tidal forcing and magnetospheric forcing; (2) the scale of Europa's potential biosphere (median figure), at which we will more particularly characterize the ocean and ice sheet and (3) finally the local scale at which we will perform life detection experiments.

This Overarching Goal can be developed into three main science objectives (Figure 1):

a) Understand Europa at the global scale as a complex system of coupled layers responding to Jupiter System forcing;

b) Define the potential habitable zone of Europa (ocean and ice shell), with a focus on the critical exchange processes of its icy surface with the sub-surface and the aqueous reservoirs below it, and with the exosphere above it;

c) Search for the signatures of a potential biosphere on the local scale. This is the culmination and converging point of our science plan: the search for and detect biosignatures. 


\section{Proposed scientific instruments:}

The implementation of the JEM science plan is based on the joint operation of two complementary platforms: a lander and a carrier/orbiter. The instrument suites proposed on each of these platforms are given in Tables 1 and 2: see [2] for their description.

The Orbiter will carry the following instruments, some of them to be operated during a minimum of 35 days simultaneously with Lander data relay, then all of them during the subsequent 3 months of orbital and descent science.

\begin{tabular}{|c|c|c|c|c|c|c|}
\hline \multicolumn{6}{|c|}{ TABLE 1: Orbital Science Platform projected required resources } & \\
\hline Facility/Instrument & $\begin{array}{c}\text { Outside the } \\
\text { vault }\end{array}$ & \multicolumn{2}{|c|}{ Inside the vault } & & & \\
\hline Core payload & - & 3.4 & 0.006 & 3.4 & 22 & 5 \\
\hline $\begin{array}{c}\text { Gass } \\
\text { Gravity Science } \\
\text { Investigation (GSI) }\end{array}$ & $\begin{array}{c}\text { Volume } \\
(\mathrm{m} 3)\end{array}$ & $\begin{array}{c}\text { Total } \\
(\mathrm{kg})\end{array}$ & $\begin{array}{c}\text { Power } \\
(\mathrm{W})\end{array}$ & TRL \\
\hline Magnetometer (MAG) & 0.1 & 0.1 & 0.001 & 0.2 & 0.4 & $8 / 9$ \\
\hline Laser Altimeter (ELA) & 11 & 9 & 0.08 & 20 & 40 & $5-6$ \\
\hline $\begin{array}{c}\text { lon Mass } \\
\text { Spectrometer + } \\
\text { Electron Spectrometer } \\
\text { (IMS/ELS) }\end{array}$ & 7 & 3 & 0.006 & 10 & 11 & 5 \\
\hline $\begin{array}{c}\text { lon and Neutral Mass } \\
\text { Spectrometer (INMS) }\end{array}$ & 3.2 & 3 & 0.006 & 6.2 & 16 & $5-6$ \\
\hline Dust Analyser (SUDA) & 7.5 & 2.6 & 0.003 & 10.1 & 9.7 & $5-6$ \\
\hline Total for core payload & 28.8 & 21.1 & 0.102 & 49,9 & 99.1 & \\
\hline $\begin{array}{c}\text { Augmentation: } \\
\text { Langmuir Probe (LP) }\end{array}$ & 1.6 & 3 & 0.004 & 4.6 & 6 & $5-6$ \\
\hline
\end{tabular}

For the lander, the current baseline is the instrument suite identified by the Europa Lander Definition Team [3]. The final payload will be determined via a competitive call for instruments. In this context the JEM team proposes that Europe could contribute with the Astrobiological Wet Laboratory (AWL) instrument package carrying three life-detection instruments working in liquid phase: Multi-probe immunoassay (MPAS); Multiparametric probe (MPP) ; VISTA (Volatiles In Situ Thermogravimetric Analyzer). Three geophysics sensors (geophone, magnetometer and laser reflector) are also strongly suggested.

Table 2. AWL Surface Science Platform instruments and required resources

\begin{tabular}{|l|c|c|c|}
\hline Facility/Instrument & Mass (kg) & Power (W) & TRL \\
\hline $\begin{array}{l}\text { AWL sensors } \\
\text { MPAS }\end{array}$ & 0.15 & 1.4 & $3-4$ \\
\cline { 2 - 4 } $\begin{array}{l}\text { AWL sensors MPP } \\
\text { AWL sensors } \\
\text { VISTA }\end{array}$ & 0.1 & 1 & $3-4$ \\
\cline { 2 - 4 } & 0.09 & 0.24 & $5-6$ \\
\hline $\begin{array}{c}\text { Total for AWL (cf. } \\
\text { 4.3.3.2) }\end{array}$ & $\begin{array}{c}11 \text { (incl. 7 for } \\
\text { shielding) }\end{array}$ & $17.4 \mathrm{Whr}$ & \\
\hline MAG & 0.6 & 0.8 & $8 / 9$ \\
\hline Laser Reflector & 0.0025 & - & - \\
\hline Geophone & 0.3 & 0.5 & $5-6$ \\
\hline
\end{tabular}




\section{Proposed mission configuration and profile}

\section{JEM flight and scientific observation sequences}

The 2 JEM platforms will perform 3 sequences of scientific observations:

A. A surface science sequence involving the lander instruments, planned to last about 35 days on a selected site;

B. An orbital science sequence involving the orbiter instruments. This sequence will first overlap in time with the surface science sequence;

$>$ C. A descent science sequence, after the end of sequence B and until Europa surface impact, to search for biomolecules in the densest layers of the exosphere.

The choice of the orbital sequences to be used successively (Table 3 ) will be the result of a trade-off in the 3-D parameter space described by the Total lonizing Dose (TID), the shielding thickness used to protect the equipment and mitigate radiation dose effects and the total Delta $\vee$ provided by the propulsion system.

\begin{tabular}{|c|c|c|c|c|c|}
\hline Sequence & $\begin{array}{l}\text { Sequence } \\
\text { name }\end{array}$ & Mission function & $\begin{array}{l}\text { Flight } \\
\text { time }\end{array}$ & $\Delta V(\mathrm{~m} / \mathrm{s})$ & $\begin{array}{l}\text { TID @ 2.5mm } \\
\text { Al }\end{array}$ \\
\hline S-1 & $\begin{array}{l}\text { Launch + } \\
\text { cruise }\end{array}$ & $\begin{array}{l}\text { Reach Jupiter } \\
\text { System }\end{array}$ & 4,9 years & 800 & $\sim$ \\
\hline S-2 & $\begin{array}{l}\mathrm{JOI}+\mathrm{PRM} \\
\text { maneuver }\end{array}$ & $\begin{array}{l}\text { Insert into the } \\
\text { Jovian system }\end{array}$ & $\begin{array}{l}6,5 \\
\text { months }\end{array}$ & 1000 & $\sim$ \\
\hline S-3 & $\begin{array}{l}\text { Jovian tour to } \\
\text { Europa vicinity }\end{array}$ & $\begin{array}{l}\text { Phase the } \\
\text { spacecraft with } \\
\text { Europa }\end{array}$ & $\begin{array}{l}9,5 \\
\text { months }\end{array}$ & 100 & $125 \mathrm{krad}$ \\
\hline S-4 & $\begin{array}{l}\text { EOI + Ejection } \\
\text { to relay orbit }\end{array}$ & $\begin{array}{l}\text { Insert into Europa, } \\
\text { release the lander, } \\
\text { reach relay orbit }\end{array}$ & & 700 & $\sim$ \\
\hline S-5 & Lander relay & $\begin{array}{l}\text { Relay and } \\
\text { downlink lander } \\
\text { data }\end{array}$ & 35 days & $\sim$ & $370 \mathrm{krad}$ \\
\hline S-6 & Relay to LEO & $\begin{array}{l}\text { Reach low-altitude } \\
\text { quasi-polar orbit }\end{array}$ & 1-3 days & $\begin{array}{l}400 \\
(\mathrm{TBC})\end{array}$ & $12 \mathrm{krad} /$ day \\
\hline S-7 & $\begin{array}{l}\text { LEO } \\
\text { operations } \\
\end{array}$ & $\begin{array}{l}\text { Support orbiter } \\
\text { science mission }\end{array}$ & 3 months & 50 & 930 krad \\
\hline S-8 & $\begin{array}{l}\text { Descent to } \\
\text { surface }\end{array}$ & & & & \\
\hline S-9 & Impact & End of mission & & & \\
\hline Total & & & 6,6 years & $\begin{array}{l}3,05 \\
\mathrm{~km} / \mathrm{s}\end{array}$ & 1,5 Mrad \\
\hline
\end{tabular}

Table 1: approximate flight time $\Delta V$ and TID (behind 2,5 $\mathrm{mm}$ finite $\mathrm{Al}$ slab shell)

\section{JEM flight and science platforms}

In its baseline configuration, the JEM flight system is composed of two platforms.

\section{The carrier/orbiter/relay platform:}

This platform will fulfil the key functions of injecting the lander stack into an Europan orbit just prior to its de-orbitation, and of relaying the lander data to Earth. It will also carry a 
focused instrument suite to perform global high-resolution measurements of the gravity, magnetic field and topography fields and of the plasma/neutral environment along Europan orbits. The proposed JEM orbiter concept is inherited from two spacecraft currently developed by ESA:

- The European Service Module (ESM) of the Orion Multi-Purpose Crew Vehicle (MPCV), from which the mechanical and propulsion bus is adapted for JEM,

- The Jupiter Icy Moons Explorer (JUICE), which provides a relevant basis for the avionics of an interplanetary mission to Jupiter.

The ESM serves as primary power and propulsion component of the Orion spacecraft. It presents several advantages for the JEM mission: it is able to carry a very heavy payload (the Orion Crew Vehicle is in the 10 tons class), it is launched on SLS, and it is developed in a NASA / ESA collaboration framework, a key asset for the JEM mission.

The JUICE spacecraft provides key assets for the other components of the JEM orbiter: a rad-hard avionics adapted to the specific constraints of an interplanetary mission and protected within a lead-shielded vault, and a power subsystem designed for LILT (Low Intensity Low Temperature) conditions.

The proposed JEM orbiter/carrier platform configuration is described in figures 2 and 3.
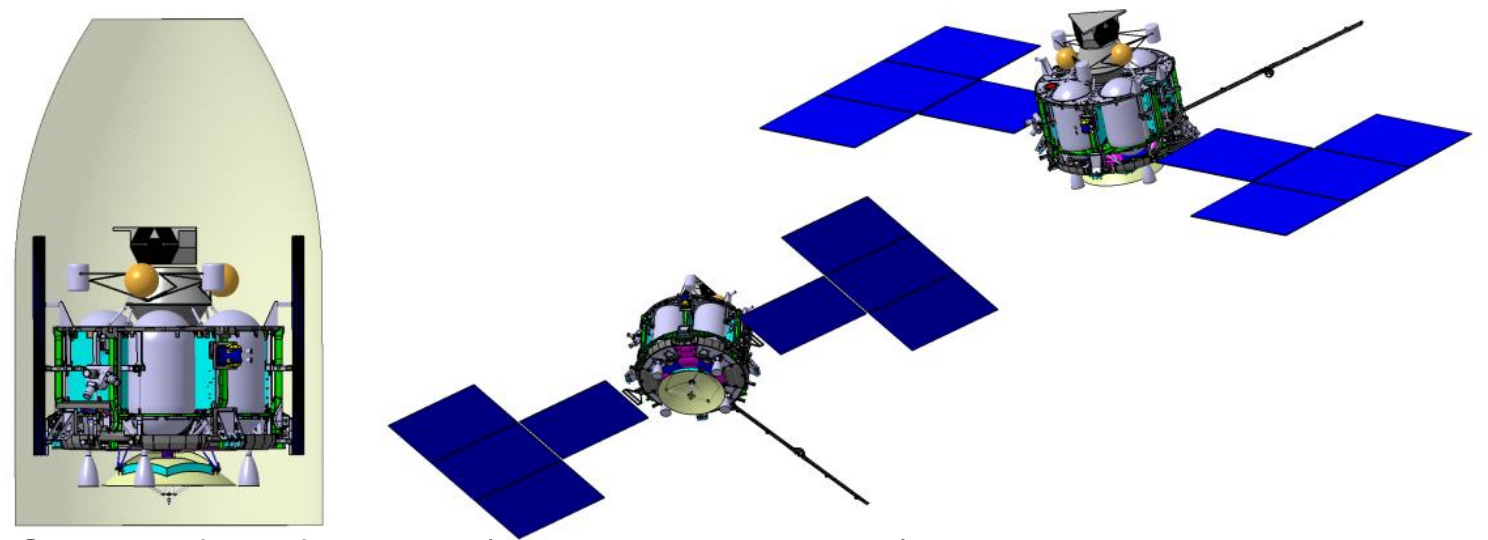

Figure 2: Spacecraft configuration (stacked and deployed)
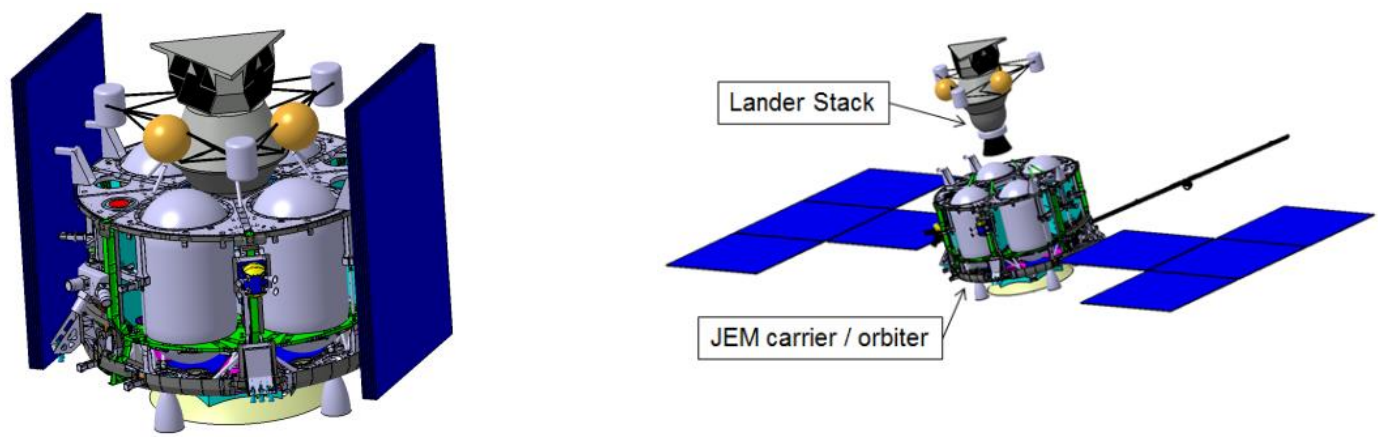

Figure 3: JEM carrier and lander interface

The JEM Lander complement.

The soft lander platform is assumed to be delivered by NASA, based on its 2017 Europa Lander study. The analysis of samples of astrobiological interest will be performed by two complementary sample analysis facilities, one devoted to the analysis of solid samples, and another one dealing with liquid samples, the Astrobiology Wet Laboratory (AWL). The two facilities will be served by a common articulated arm. In addition to astrobiology investigations, the lander will also operate a geophysics station for the study of planetary fields and of the properties of the surface ice, and for sounding of the sub-surface. 


\section{The Astrobiology Wet Laboratory (AWL)}

We envisage two accommodation options for the AWL (Figure 4): inside the lander or at the surface. The latter option requires the arm hold the instrument and deposit it on the surface. The reason for selecting one of other option could be based on the arm design constrains but also the biochemical cleanliness conditions. The AWL detects large organics molecules (proteins, lipids, etc.). To avoid false positives the level of biochemical cleanliness of the arm solid sampler should be stricter than if it only supplies samples to a GCMS or a Raman spectrometer. If the AWL works at the surface, it has its own sampler. If it operates inside the lander it only has a module to liquefy the sample. The AWL could also host the magnetometer with a small increase of mass (deployment boom, sensor head and electronic). If it is on the lander it could include the thermogravimeter, making the proposed European contribution an independent package with clear interfaces with the NASA lander.

AWL/S description: In the AWL/S option it is composed of: i) a Sample Acquisition Module in charge of making a $10 \mathrm{~cm}$ hole to take a liquid sample, ii) the Data Processing Unit; iii) a Power Unit; iv) a Communication Unit. An external structure support allows one to deploy the AWL with the lander manipulator. For the Sample Acquisition Module (SAM), we have evaluated different alternatives. The most promising option is the use of a drilling system based on laser [4]. The water sample is taken in two steps: i) the first 5 $\mathrm{cm}$ of ice are sublimated by the laser; ii) the tube is moved down by a pneumatic actuator and once in contact penetrates by $5 \mathrm{~cm}$ in the ice. The tube is pressurized and heated to provide conditions in which the water is stable. At this moment the sample is sucked by a syringe (controlled by a spring) to fill the sample deposit.

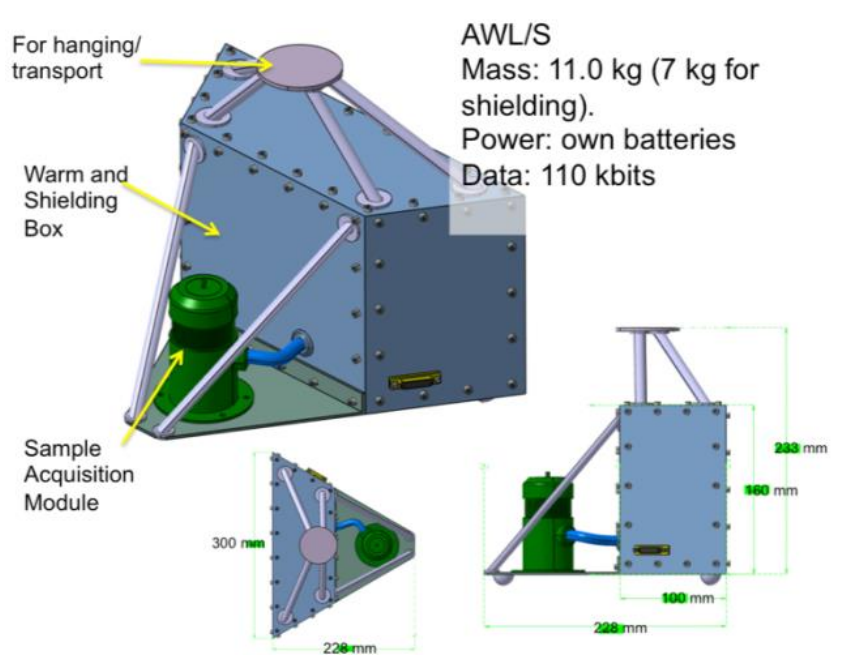

Figure 4: AWL/S mechanical configuration concept. A support structure allows it to be handled by the lander arm. A box protects the electronics, MAP and MPP. The isolation lid, below SAM, has a lateral movement to be open.

From this deposit the instruments are filled. A single pressurized deposit (nitrogen TBC) is used for tube movement and pressurization. The most critical components of the AWL/S are the batteries. They are the heaviest element and need to be maintained above a determined temperature to maintain their performances. For radiation protection, the Warm and Shielding Box has a thickness of $18 \mathrm{~mm} \mathrm{Al}$ to allow the use of space standard components. Figure 5 shows the AWL mechanical configuration. A warm and shielding box (WSB) is used to maintain the operational temperature and protect all the electronics for radiation. The WSB will guarantee by design bio-cleanliness after integration. The SAM will have an isolation lid that will be closed once at the end of the integration to maintain the biological cleanliness. An opening protected with an EPA filter will help the decompression during launch. The external structure supports the magnetometer boom and allows hanging to the lander articulated arm. This configuration allows ejection from the lander if for some scientific reason it was recommended to explore some site far from it. The AWL side could be equipped with small airbags and following a similar concept implemented in the Pathfinder lander, a set of petals could guarantee that it stays always in the vertical orientation. 
AWL/L description: The main difference with the AWL/L is the SAM, which in this case is reduced to a module to liquefy the sample and has no batteries, making the Power Unit much simpler. The process for obtaining the liquid sample is similar to the one proposed for the AWL/S.

\section{Proposed international collaboration scheme:}

The design and planning of JEM will take advantage of the heritage of several missions to the Jupiter System to be flown by ESA and NASA before 2035: Juno (currently in operation), Europa Clipper and JUICE. NASA's Europa Clipper will provide a first assessment of Europa's habitability and the data returned from its 45 Europa fly-bys will provide the basis for a lander mission. In terms of Europa's geophysics and internal structure, flying a Europa orbiter mission in polar orbit, even for a limited duration, will provide a strong additional contribution to the characterization of Europa's habitability.

Coming after these previous missions, JEM will be in ideal position to perform a comprehensive description of Europa as a habitable world and to search for life at its surface. The major scientific and technological challenges of such an endeavor can be met if NASA (with its long and deep investments in the science and technology of a Europa Lander) and ESA (with its expertise on the Jupiter system gained with JUICE) join forces to search for life at Europa.

We propose the following share of responsibilities between ESA and NASA: (1) The lander will be provided and operated by NASA with the support of ESA; (2) NASA will study with ESA the possibility of deploying from that platform a small ESA-provided « Astrobiology Wet Laboratory (AWL)» as an option; (3) ESA will take a major responsibility in the delivery of the carrier/orbiter/relay platform, ranging from the delivery of the full platform to the delivery of an integrated "science investigation platform » and of critical subsystems; (4) The proposed selection of scientific investigations on the different flight elements will be validated by ESA for the carrier/orbiter and by NASA for the lander.

Acknowledgements: The authors received support from the sponsors of their home institutions during the development of their projects. At IRAP, Toulouse, MB and NA acknowledge the support of CNRS, University Toulouse III - Paul Sabatier and CNES. At CAB, Madrid, OPB and JGE acknowledge the support of INTA and Spanish MINECO project ESP2014-55811-C2-1-P and ESP2017-89053-C2-1-P and the AEI project MDM-2017-0737 Unidad de Excelencia "María de Maeztu.". The JEM proposing team would like to express its gratitude to the mission design team of Airbus Defence and Space in Toulouse and to the PASO team of CNES for their support in the study of the JEM mission concept.

\section{BIBLIOGRAPHY}

[1] Blanc et al., Accepted for publication, Planet. Space. Sci. (2020)

[2] Blanc et al. (2019), White Paper submitted to ESA's Voyage 2050 call for ideas.

[3] Hand, K. et al (2017), NASA Europa Lander SDT report.

[4] Sakurai T. et al., (2016). Cold Regions Science and technology 121 
